\title{
Adaptive Integral Sliding Mode Stabilization of Nonholonomic Drift-Free Systems
}

\author{
Waseem Abbasi ${ }^{1,2}$ and Fazal ur Rehman ${ }^{1}$ \\ ${ }^{1}$ Department of Electrical Engineering, Capital University of Science and Technology (CUST), Kahuta Road, \\ Express Highway, Islamabad 44000, Pakistan \\ ${ }^{2}$ Department of Electrical Engineering, The University of Lahore (UOL), Japan Road, Express Highway, \\ Islamabad 44000, Pakistan \\ Correspondence should be addressed to Waseem Abbasi; waseemabbasi97@gmail.com
}

Received 23 June 2016; Revised 28 September 2016; Accepted 19 October 2016

Academic Editor: Muhammad N. Akram

Copyright (c) 2016 W. Abbasi and F. U. Rehman. This is an open access article distributed under the Creative Commons Attribution License, which permits unrestricted use, distribution, and reproduction in any medium, provided the original work is properly cited.

\begin{abstract}
This article presents adaptive integral sliding mode control algorithm for the stabilization of nonholonomic drift-free systems. First the system is transformed, by using input transform, into a special structure containing a nominal part and some unknown terms which are computed adaptively. The transformed system is then stabilized using adaptive integral sliding mode control. The stabilizing controller for the transformed system is constructed that consists of the nominal control plus a compensator control. The compensator control and the adaptive laws are derived on the basis of Lyapunov stability theory. The proposed control algorithm is applied to three different nonholonomic drift-free systems: the unicycle model, the front wheel car model, and the mobile robot with trailer model. The controllability Lie algebra of the unicycle model contains Lie brackets of depth one, the model of a front wheel car contains Lie brackets of depths one and two, and the model of a mobile robot with trailer contains Lie brackets of depths one, two, and three. The effectiveness of the proposed control algorithm is verified through numerical simulations.
\end{abstract}

\section{Introduction}

Designing feedback control laws for the stabilization of mechanical control systems has been an interesting subject for researchers in the field of control theory. These systems have attracted intensive attention from the control community because of their wide practical applications in robotics, industry, and automobiles. Due to mechanical design and configuration, these systems are classified into two categories: holonomic and nonholonomic. In holonomic systems, the control input degrees are equal to total degrees of freedom, whereas, nonholonomic systems have less controllable degrees of freedom as compared to total degrees of freedom and have restricted mobility due to the presence of nonholonomic constraints. Roger Brockett showed that the nonholonomic systems cannot be stabilized by continuous static state feedback laws [1]. Later on Murray et al. showed that the dependence of the stabilizing control on time is essential [2].
To solve this problem, different control approaches have been presented in the literature. A detailed survey of stabilization of nonholonomic systems can be found in [3] and a survey of underactuated mechanical systems is given in [4]. In the literature, several control techniques have been developed for stabilization of nonholonomic systems. Some of these include discontinuous time-invariant techniques [5-8], time-varying techniques [9-12], adaptive techniques $[13,14]$, and sliding mode control technique [15-20]. Sliding mode control (SMC) is a special nonlinear control technique. The objective of the SMC technique is to force the system states to a certain surface, known as the sliding manifold. Once the surface is reached, the system is forced to remain on it thereafter.

The main disadvantage of the SMC is the requirement of discontinuous control law across the sliding manifold. In practical systems, this leads to an undesirable phenomenon called chattering. The closed loop dynamics of the system in 
SMC depends only on the design parameters of the switching sliding manifold. Sliding mode control also offers several advantages such as simplicity, fast response, and robustness to external disturbance and parameter variation.

Our objective in this article is to propose a scheme for the construction of stabilizing control for nonholonomic mechanical systems. The suggested sliding mode controller can stabilize systems, which do not fulfill Brockett's necessary conditions, as the sliding mode control is inherently discontinuous. Since sliding mode control is insensitive towards model errors, parametric uncertainties, and other disturbances; therefore, it is extensively used. Sliding surface will show system's behavior when the system reaches the sliding manifold [21-24].

The integral sliding mode control guarantees the robustness of the motion in the whole state space $[25,26]$ because of eliminating the reaching phase. Since the reaching phase is eliminated, therefore the robustness of the system can be guaranteed throughout the system response, starting from the initial time instance. The integral sliding mode control combines the nominal control that stabilizes the nominal system and a discontinuous control that rejects the uncertainty.

The control algorithm presented in this paper is general and applicable to a large class of nonholonomic control systems without drift. The proposed algorithm is applied to three different nonholonomic drift-free systems: the unicycle model, the front wheel car model, and the mobile robot with trailer model. The effectiveness of the proposed algorithm is verified through numerical simulations.

The rest of the article is organized as follows. Section 2 presents problem formulation. Section 3 presents the proposed control methodology in its general form. Section 4 presents application examples of the unicycle model, the front wheel car model, and the car with trailer model. Section 5 presents simulation results for the application examples, and finally Section 6 concludes the paper.

\section{Problem Formulation}

2.1. Mathematical Model of Nonholonomic System. The kinematic model for a drift-free nonholonomic system is given as

$$
\dot{x}=\sum_{i=1}^{m} G_{i}(x) u_{i}, \quad x \in \mathfrak{R}^{n},
$$

where $G_{i}(x)$ are linearly independent vector fields on $\mathfrak{R}^{n}, u_{i}$ are locally bounded in $t$, and piece-wise continuous control functions are defined on the interval $[0, \infty)$. These systems are difficult to control as revealed by the fact that linearization of system (1) is uncontrollable. The most difficult issue from a theoretical viewpoint is the design of feedback laws that can stabilize these systems about an equilibrium position.

2.2. Problem Statement. Given a desired set point $x_{\mathrm{des}} \in \mathfrak{R}^{n}$, construct a feedback strategy in presence of the control $u_{i}$ : $\mathfrak{R}^{n} \rightarrow \mathfrak{R}, i=1,2, \ldots, m$, so that the desired set point $x_{\mathrm{des}}$ is an attractive set for $(1)$, such that $x\left(t ; 0, x_{0}\right) \rightarrow x_{\mathrm{des}}$, as $t \rightarrow \infty$ for any initial condition.

Generally, by appropriate translation of coordinate system, $x_{\text {des }}=0$ can be achieved.
2.3. Some Assumptions. For steering control problem, the systems described by (1) must satisfy the following conditions:

(P1) The vector fields $G_{1}(x), \ldots, G_{m}(x)$ are linearly independent.

(P2) System (1) satisfies the Lie algebra rank condition (LARC) for accessibility, where Lie algebra, $L\left(G_{1}, \ldots, G_{m}\right)(x)$, spans $\Re^{n}$ at each point $x \in \mathfrak{R}^{n}$.

\section{The Proposed Control Algorithm}

Step 1. Write system (1) in the following form:

$$
\begin{gathered}
\dot{x}_{1}=g_{1}(x, u), \\
\dot{x}_{2}=g_{2}(x, u), \\
\vdots \\
\dot{x}_{n-1}=g_{n-1}(x, u), \\
\dot{x}_{n}=g_{n}(x, u),
\end{gathered}
$$

where $g_{i}: \mathfrak{R}^{n} \times \mathfrak{R}^{m} \rightarrow \mathfrak{R}$ are nonlinear functions.

Step 2. Using the input transformation, transform system (2) into the following form:

$$
\begin{gathered}
\dot{x}_{1}=h_{1}(x), \\
\dot{x}_{2}=h_{2}(x), \\
\vdots \\
\dot{x}_{n-1}=h_{n-1}(x), \\
\dot{x}_{n}=v,
\end{gathered}
$$

where $h_{i}: \Re^{n} \times \mathfrak{R}^{m} \rightarrow \mathfrak{R}$ are nonlinear function and $v$ is the new input.

After some manipulation, system (3) can be rewritten as

$$
\begin{gathered}
\dot{x}_{1}=x_{2}+F_{1}, \\
\dot{x}_{2}=x_{3}+F_{2}, \\
\vdots \\
\dot{x}_{n-1}=x_{n}+F_{n-1}, \\
\dot{x}_{n}=v,
\end{gathered}
$$

where $F_{i}=-x_{i+1}+h_{i}(x)$.

Step 3. Assume that $F_{i}$ are uncertainties in the system. Let $\widehat{F}_{i}, i=1, \ldots, n$ be an estimate of $F_{i}, i=1, \ldots, n-1$, respectively. Apply the function approximation technique [27] to represent $F_{i}$ and their estimates $\widehat{F}_{i}$ as $F_{i}=w_{i}^{T} \varphi_{i}(t)$ and $\widehat{F}_{i}=\widehat{w}_{i}^{T} \varphi_{i}(t)$.

$\varphi_{i}(t)=\left[\begin{array}{llll}\varphi_{i 1}(t) & \varphi_{i 2}(t) & \cdots & \varphi_{i n}(t)\end{array}\right]^{T}$ is the function of basis vector and $w_{i}=\left[\begin{array}{llll}w_{i 1} & w_{i 2} & \cdots & w_{i n}\end{array}\right]^{T}$ is a vector of 
weightings. Let $\widehat{w}_{i}=\left[\begin{array}{llll}\widehat{w}_{i 1} & \widehat{w}_{i 2} & \cdots & \widehat{w}_{i n}\end{array}\right]^{T}$ be estimate of $w_{i}=\left[\begin{array}{llll}w_{i 1} & w_{i 2} & \cdots & w_{i n}\end{array}\right]^{T}$. Therefore, we can estimate $F_{i}$ by estimating the weight vector $w_{i}$; that is, $\widehat{F}_{i}=\widehat{w}_{i}^{T} \varphi_{i}(t)$. Define $\widetilde{w}_{i}=w_{i}-\widehat{w}_{i}$; then system (4) can be written as

$$
\begin{gathered}
\dot{x}_{1}=x_{2}+\widehat{w}_{1}^{T} \varphi_{1}(t)+\widetilde{w}_{1}^{T} \varphi_{1}(t), \\
\dot{x}_{2}=x_{3}+\widehat{w}_{2}^{T} \varphi_{1}(t)+\widetilde{w}_{2}^{T} \varphi_{1}(t), \\
\vdots \\
\dot{x}_{n-1}=x_{n}+\widehat{w}_{n-1}^{T} \varphi_{n-1}(t)+\widetilde{w}_{n-1}^{T} \varphi_{n-1}(t), \\
\dot{x}_{n}=v .
\end{gathered}
$$

Step 4. Choose the nominal system for (5) as

$$
\begin{gathered}
\dot{x}_{1}=x_{2}, \\
\dot{x}_{2}=x_{3}, \\
\vdots \\
\dot{x}_{n-1}=x_{n}, \\
\dot{x}_{n}=v_{0} .
\end{gathered}
$$

Step 5. Define the sliding surface for nominal system (6) as

$$
s_{0}=x_{1}+\sum_{i=2}^{n-1} c_{i} x_{i}+x_{n}
$$

where $c_{i}>0$ are chosen in such a way that $s_{0}$ becomes Hurwitz polynomial. Then

$$
\dot{s}_{0}=\dot{x}_{1}+\sum_{i=2}^{n-1} c_{i} \dot{x}_{i}+\dot{x}_{n}=x_{2}+\sum_{i=2}^{n-1} c_{i} x_{i+1}+v_{0} .
$$

Choose

$$
v_{0}=-x_{2}-\sum_{i=2}^{n-1} c_{i} x_{i+1}-k \operatorname{sign}\left(s_{0}\right), \quad k>0 .
$$

We have $\dot{s}_{0}=-k \operatorname{sign}\left(s_{0}\right)$. Therefore, nominal system (6) is asymptotically stable.

Step 6. Define the sliding surface for system (5) as

$$
s=s_{0}+z=x_{1}+\sum_{i=2}^{n-1} c_{i} x_{i}+x_{n}+z,
$$

where $z$ is an integral term. To avoid the reaching phase, choose $z(0)$ such that $s(0)=0$. Choose $v=v_{0}+v_{s}$, where $v_{0}$ is the nominal input and $v_{s}$ is compensator term. Then

$$
\begin{aligned}
\dot{s}= & \dot{x}_{1}+\sum_{i=2}^{n-1} c_{i} \dot{x}_{i}+\dot{x}_{n}+\dot{z} \\
= & x_{2}+\widehat{w}_{i}^{T} \varphi_{1}(t)+\widetilde{w}_{i}^{T} \varphi_{2}(t) \\
& +\sum_{i=2}^{n-1} c_{i}\left\{x_{i+1}+\widehat{w}_{i}^{T} \varphi_{i}(t)+\widetilde{w}_{i}^{T} \varphi_{i}(t)\right\}+v_{0}+v_{s}+\dot{z}
\end{aligned}
$$

$$
\begin{gathered}
=x_{2}+\sum_{i=2}^{n-1} c_{i} x_{i+1}+v_{0}+v_{s}+\dot{z} \\
+\sum_{i=1}^{n-1}\left\{\widehat{w}_{i}^{T} \varphi_{i}(t)+\widetilde{w}_{i}^{T} \varphi_{i}(t)\right\},
\end{gathered}
$$

where $c_{1}=1$.

Step 7. Choose a Lyapunov function as

$$
V=\frac{1}{2} s^{2}+\frac{1}{2} \sum_{i=1}^{n-1} \widetilde{w}_{i}^{T} \widetilde{w}_{i}
$$

Design the adaptive laws for $\widetilde{w}_{i} \& \widehat{w}_{i}, i=1, \ldots, n$ and compute $v_{s}$ such that $\dot{V}<0$.

Theorem 1. Choose a Lyapunov function as

$$
V=\frac{1}{2} s^{2}+\frac{1}{2} \sum_{i=1}^{n-1} \widetilde{w}_{i}^{T} \widetilde{w}_{i}
$$

The following adaptive laws for $\widetilde{w}_{i}$ \& $\widehat{w}_{i}$ and the value of $v_{s}$ will guarantee the time derivative of $V$ in (13) to be strictly negative (i.e., $\dot{V}<0$ )

$$
\begin{aligned}
\dot{z} & =-x_{2}-\sum_{i=2}^{n-1} c_{i} x_{i+1}-v_{0}, \\
v_{s} & =-\sum_{i=1}^{n-1} c_{i} \widehat{w}_{i}^{T} \varphi_{i}(t)-k s, \\
\dot{\tilde{w}}_{i} & =-c_{i} s \varphi_{i}(t)-k_{i} \widetilde{w}_{i}, \\
\dot{\vec{w}}_{i} & =-\dot{\tilde{w}}_{i}
\end{aligned}
$$

where $k$ and $k_{i}>0, \quad i=1, \ldots, n-1$.

Proof. Since

$$
\begin{aligned}
\dot{V} & =s \dot{s}+\sum_{i=1}^{n-1} \widetilde{w}_{i}^{T} \dot{\tilde{w}}_{i}=s\left\{x_{2}+\widehat{w}_{1}^{T} \varphi_{1}(t)+\widetilde{w}_{1}^{T} \varphi_{1}(t)\right. \\
& \left.+\sum_{i=2}^{n-1} c_{i}\left\{x_{i+1}+\widehat{w}_{i}^{T} \varphi_{i}(t)+\widetilde{w}_{i}^{T} \varphi_{i}(t)\right\}+v_{0}+v_{s}+\dot{z}\right\} \\
& +\sum_{i=1}^{n-1} \widetilde{w}_{i}^{T} \dot{\tilde{w}}_{i}=s\left\{x_{2}+\sum_{i=1}^{n-1}\left\{c_{i} x_{i+1}+c_{i} \widehat{w}_{i}^{T} \varphi_{i}(t)\right\}+v_{0}\right. \\
& \left.+v_{s}+\dot{z}\right\}+\sum_{i=1}^{n-1} \widetilde{w}_{i}^{T}\left\{\dot{\tilde{w}}_{i}+c_{i} s \varphi_{i}(t)\right\},
\end{aligned}
$$

by using

$$
\begin{gathered}
\dot{z}=-x_{2}-\sum_{i=2}^{n-1} c_{i} x_{i+1}-v_{0}, \\
v_{s}=-\sum_{i=1}^{n-1} c_{i} \widehat{w}_{i}^{T} \varphi_{i}(t)-k s,
\end{gathered}
$$




$$
\begin{aligned}
& \dot{\widetilde{w}}_{i}=-c_{i} s \varphi_{i}(t)-k_{i} \widetilde{w}_{i}, \\
& \dot{\widehat{w}}_{i}=-\dot{\widetilde{w}}_{i},
\end{aligned}
$$

where $k$ and $k_{i}>0, i=1, \ldots, n-1$, we have

$$
\dot{V}=-k s^{2}-\sum_{i=1}^{n-1} k_{i} \widetilde{w}_{i}^{T} \widetilde{w}_{i} .
$$

Choosing

$$
k_{n}=\min \left(k, k_{1}, \ldots, k_{n-1}\right) \text {, }
$$

we have

$$
\dot{V} \leq-k_{n}\left(s^{2}+\sum_{i=1}^{n-1} \widetilde{w}_{i}^{T} \widetilde{w}_{i}\right)
$$

Using the chosen Lyapunov function

$$
V=\frac{1}{2} s^{2}+\frac{1}{2} \sum_{i=1}^{n-1} \widetilde{w}_{i}^{T} \widetilde{w}_{i},
$$

we can write

$$
\begin{aligned}
& \dot{V} \leq-2 k_{n} V, \\
& \dot{V} \leq-\alpha V^{\beta},
\end{aligned}
$$

where $\alpha=2 k_{n}$ and $\beta=1$.

From this we conclude that $s \& \widetilde{w}_{i} \rightarrow 0, i=1, \ldots, n$. Since $s \rightarrow 0$, therefore $x \rightarrow 0$.

In the following section we illustrate the above algorithm by applying it to three different nonholonomic drift-free systems.

\section{Application Examples}

4.1. The Unicycle Model. A unicycle model or a two-wheel car model, shown in Figure 1, is basically a three-dimensional nonholonomic system having two inputs and three states with depth-one Lie bracket. A two-wheel car kinematic model is defined as [12]

$$
\left[\begin{array}{l}
\dot{\theta} \\
\dot{x} \\
\dot{y}
\end{array}\right]=\left[\begin{array}{l}
1 \\
0 \\
0
\end{array}\right] u_{1}+\left[\begin{array}{c}
0 \\
\cos \theta \\
\sin \theta
\end{array}\right] u_{2} \text {. }
$$

Introducing a new set of state variables $x \stackrel{\text { def }}{=}\left[x_{1}, x_{2}, x_{3}\right]^{T}=$ $[\theta, x, y]^{T}$ the kinematics model (22) can be written as

$$
\left[\begin{array}{l}
\dot{x}_{1} \\
\dot{x}_{2} \\
\dot{x}_{3}
\end{array}\right]=\left[\begin{array}{l}
1 \\
0 \\
0
\end{array}\right] u_{1}+\left[\begin{array}{c}
0 \\
\cos x_{1} \\
\sin x_{1}
\end{array}\right] u_{2}
$$

or

$$
\dot{x}=G_{1}(x) u_{1}+G_{2}(x) u_{2}, \quad x \in \mathfrak{R}^{3},
$$

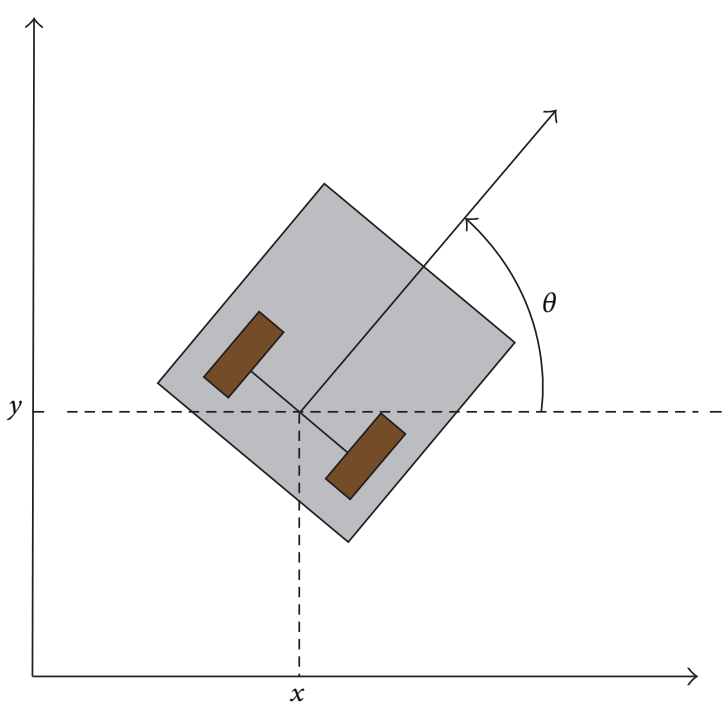

Figure 1: The unicycle model.

where

$$
\begin{aligned}
& G_{1}(x)=\left[\begin{array}{l}
1 \\
0 \\
0
\end{array}\right], \\
& G_{2}(x)=\left[\begin{array}{c}
0 \\
\cos x_{1} \\
\sin x_{1}
\end{array}\right] .
\end{aligned}
$$

The kinematics model (22) satisfies the following assumptions:

(P1) The vector fields $G_{1}(x)$ and $G_{2}(x)$ are linearly independent.

(P2) System (24) satisfies the Lie algebra rank condition (LARC) for accessibility, where the Lie algebra, $L\left(G_{1}, G_{2}\right)(x)$, spans $\mathfrak{R}^{3}$ at each point $x \in \mathfrak{R}^{3}$.

To verify property (P2), it is sufficient to calculate the following Lie bracket of $G_{1}(x)$ and $G_{2}(x)$ :

$$
G_{3}(x) \stackrel{\text { def }}{=}\left[G_{1}, G_{2}\right](x)=\left[\begin{array}{c}
0 \\
-\sin x_{1} \\
\cos x_{1}
\end{array}\right] .
$$

Then the LARC condition, namely, $\operatorname{span}\left(G_{1}, G_{2}, G_{3}\right)(x)=$ $\mathfrak{R}^{3}, \forall x \in \mathfrak{R}^{3}$, is satisfied.

\subsubsection{Application of the Proposed Algorithm to the Unicycle Model}

Step 1. The unicycle model given (24) can be rewritten as

$$
\begin{aligned}
& \dot{x}_{1}=u_{1}, \\
& \dot{x}_{2}=\cos x_{1} u_{2}, \\
& \dot{x}_{3}=\sin x_{1} u_{2} .
\end{aligned}
$$


Step 2. Choose $u_{1}=v$ and $u_{2}=x_{3} / \cos x_{1}$, where, $x_{1} \neq \pi / 2$; then system (27) becomes

$$
\begin{aligned}
& \dot{x}_{1}=v, \\
& \dot{x}_{2}=x_{3}, \\
& \dot{x}_{3}=x_{3} \tan x_{1} .
\end{aligned}
$$

After some manipulation the above-mentioned system can be written as

$$
\begin{aligned}
& \dot{x}_{2}=x_{3}, \\
& \dot{x}_{3}=x_{1}+F, \\
& \dot{x}_{1}=v,
\end{aligned}
$$

where $F=-x_{1}+x_{3} \tan x_{1}$.

Step 3. Assume $F$ as an uncertainty and let $\widehat{F}$ be an estimate of $F$. The estimate of $F$ by function approximating technique [27] is $F=w^{T} \varphi$. Then $\widehat{F}=\widehat{w}^{T} \varphi$ and system (29) can be written as

$$
\begin{aligned}
& \dot{x}_{2}=x_{3}, \\
& \dot{x}_{3}=x_{1}+\widehat{w}^{T} \varphi+\widetilde{w}^{T} \varphi, \\
& \dot{x}_{1}=v .
\end{aligned}
$$

Step 4. Choose the nominal system for (30) as

$$
\begin{aligned}
& \dot{x}_{2}=x_{3}, \\
& \dot{x}_{3}=x_{1}, \\
& \dot{x}_{1}=v_{0} .
\end{aligned}
$$

Step 5. Define the sliding surface for nominal system (31) as

$$
s_{0}=x_{2}+2 x_{3}+x_{1}
$$

Then

$$
\dot{s}_{0}=\dot{x}_{2}+2 \dot{x}_{3}+\dot{x}_{1}=x_{3}+2 x_{1}+v_{0}
$$

By choosing

$$
v_{0}=-x_{3}-2 x_{1}-k \operatorname{sign}\left(s_{0}\right), \quad k>0,
$$

we have

$$
\dot{s}_{0}=-k \operatorname{sign}\left(s_{0}\right) .
$$

Therefore, nominal system (31) is asymptotically stable.

Step 6. Define the sliding surface for system (30) as

$$
s=s_{0}+z=x_{2}+2 x_{3}+x_{1}+z .
$$

Choose $v=v_{0}+v_{s}$.

Then

$$
\begin{aligned}
\dot{s} & =\dot{x}_{1}+2 \dot{x}_{3}+\dot{x}_{2}+\dot{z} \\
& =x_{3}+2 x_{2}+2 \widehat{w}^{T} \varphi+2 \widetilde{w}^{T} \varphi+v_{0}+v_{s}+\dot{z}
\end{aligned}
$$

Step 7. The adaptive laws for $\widetilde{w}, \widehat{w}$ and the value of $v_{s}$ are as follows:

$$
\begin{aligned}
\dot{z} & =-x_{3}-2 x_{2}-v_{0}, \\
v_{s} & =-2 \widehat{w}^{T} \varphi-k s, \\
\dot{\widetilde{w}} & =-2 s \varphi-k_{1} \widetilde{w}, \\
\dot{\widehat{w}} & =-\dot{\widetilde{w}},
\end{aligned}
$$

where $k$ and $k_{1}>0$.

Give

$$
\dot{V}=-k s^{2}-k_{1} \widetilde{w}^{T} \widetilde{w}
$$

where $V=(1 / 2) s^{2}+(1 / 2) \widetilde{w}^{T} \widetilde{w}$.

Choosing

$$
k_{2}=\min \left(k, k_{1}\right) \text {, }
$$

we have

$$
\dot{V} \leq-k_{2}\left(s^{2}+\widetilde{w}^{T} \widetilde{w}\right)
$$

Using the chosen Lyapunov function we can write

$$
\begin{aligned}
& \dot{V} \leq-2 k_{2} V, \\
& \dot{V} \leq-\alpha V^{\beta},
\end{aligned}
$$

where $\alpha=2 k_{2}$ and $\beta=1$.

From this we conclude that $s \& \widetilde{w} \rightarrow 0$. Since $s \rightarrow 0$, therefore $x \rightarrow 0$.

Simulation results are shown in Figure 4.

4.2. The Front Wheel Car Model. A front wheel car model, shown in Figure 2, is basically a four-dimensional nonholonomic system having two inputs and four states with depthtwo Lie bracket. A front wheel car kinematic model [6] can be defined as

$$
\left[\begin{array}{c}
\dot{\psi} \\
\dot{x} \\
\dot{\theta} \\
\dot{y}
\end{array}\right]=\left[\begin{array}{l}
1 \\
0 \\
0 \\
0
\end{array}\right] u_{1}+\left[\begin{array}{c}
0 \\
\cos \theta \\
\frac{1}{l} \tan \psi \\
\sin \theta
\end{array}\right] u_{2} .
$$

Assuming that $l=1$ and introducing a new set of state variables $x \stackrel{\text { def }}{=}\left(x_{1}, x_{2}, x_{3}, x_{4}\right)=(\psi, x, y, \theta)$ the kinematics model (43) can be written as

$$
\left[\begin{array}{l}
\dot{x}_{1} \\
\dot{x}_{2} \\
\dot{x}_{3} \\
\dot{x}_{4}
\end{array}\right]=\left[\begin{array}{l}
1 \\
0 \\
0 \\
0
\end{array}\right] u_{1}+\left[\begin{array}{c}
0 \\
\cos x_{4} \\
\sin x_{4} \\
\tan x_{1}
\end{array}\right] u_{2}
$$

or

$$
\dot{x}=G_{1}(x) u_{1}+G_{2}(x) u_{2}, \quad x \in \mathfrak{R}^{4},
$$

where $G_{1}(x)=\left[\begin{array}{l}1 \\ 0 \\ 0 \\ 0\end{array}\right]$ and $G_{2}(x)=\left[\begin{array}{c}0 \\ \cos x_{4} \\ \sin x_{4} \\ \tan x_{1}\end{array}\right]$. 


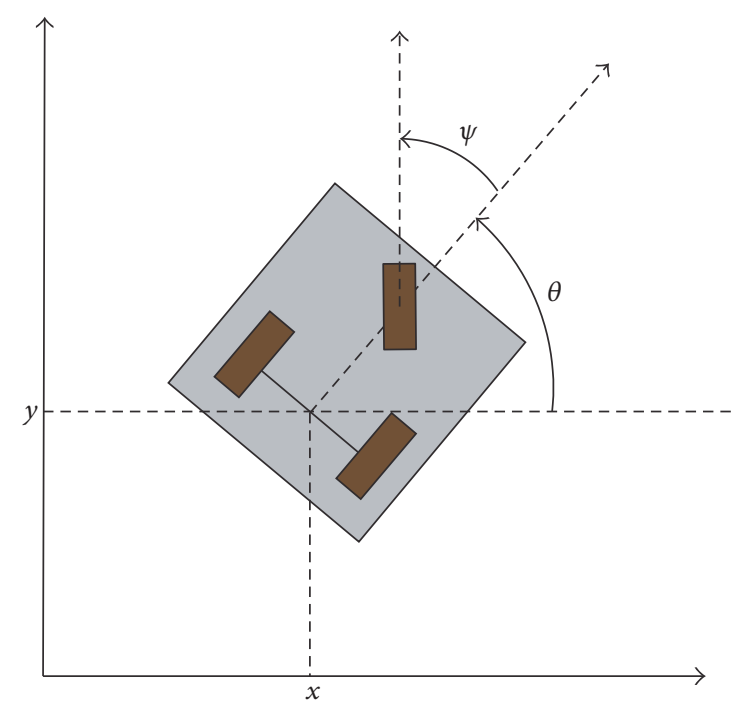

FIgURE 2: The front wheel car model. tions:

The kinematics model (45) satisfies the following assump-

(P1) The vector fields $G_{1}(x)$ and $G_{2}(x)$ are linearly independent.

(P2) System (45) satisfies the Lie algebra rank condition (LARC) for accessibility, where the Lie algebra, $L\left(G_{1}, G_{2}\right)(x)$, spans $\mathfrak{R}^{4}$ at each point $x \in \mathfrak{R}^{4}$.

To verify property (P2), it is sufficient to calculate the following Lie brackets of $G_{1}(x) \& G_{2}(x)$ :

$$
\begin{aligned}
G_{3}(x) \stackrel{\text { def }}{=}\left[G_{1}, G_{2}\right](x)=\left[\begin{array}{c}
0 \\
0 \\
0 \\
\left(\sec x_{1}\right)^{2}
\end{array}\right], \\
G_{4}(x) \stackrel{\text { def }}{=}\left[G_{2}, G_{3}\right](x)=\left[\begin{array}{c}
0 \\
-\sin x_{4}\left(\sec x_{1}\right)^{2} \\
\cos x_{4}\left(\sec x_{1}\right)^{2} \\
0
\end{array}\right],
\end{aligned}
$$

which satisfy the LARC condition: $\operatorname{span}\left(G_{1}, G_{2}, G_{3}, G_{4}\right)(x)=$ $\mathfrak{R}^{4}, \forall x \in \mathfrak{R}^{4}$.

\subsubsection{Application of the Proposed Algorithm to the Front Wheel Car Model}

Step 1. The front wheel car model as given in (45) can be rewritten as

$$
\begin{aligned}
& \dot{x}_{1}=u_{1}, \\
& \dot{x}_{2}=\cos x_{4} u_{2}, \\
& \dot{x}_{3}=\sin x_{4} u_{2}, \\
& \dot{x}_{4}=\tan x_{1} u_{2} .
\end{aligned}
$$

Step 2. Choose $u_{1}=v$ and $u_{2}=x_{3} / \cos x_{4}$, where $x_{4} \neq \pi / 2$, and then system (47) becomes

$$
\begin{aligned}
& \dot{x}_{1}=v, \\
& \dot{x}_{2}=x_{3}, \\
& \dot{x}_{3}=x_{3} \tan x_{4}, \\
& \dot{x}_{4}=x_{3} \tan x_{1} \sec x_{4},
\end{aligned}
$$

which can be rewritten as

$$
\begin{aligned}
& \dot{x}_{2}=x_{3}, \\
& \dot{x}_{3}=x_{4}+F_{3}, \\
& \dot{x}_{4}=x_{1}+F_{4}, \\
& \dot{x}_{1}=v,
\end{aligned}
$$

where

$$
\begin{aligned}
& F_{3}=-x_{4}+x_{3} \tan x_{4}, \\
& F_{4}=-x_{1}+x_{3} \tan x_{1} \sec x_{4} .
\end{aligned}
$$

Step 3. Treat $F_{i}, i=3,4$ as uncertainties and let $\widehat{F}_{i}, i=3,4$ be an estimate of $F_{i}, i=3,4$, respectively. Using function approximation technique [27], we can approximate $F_{i}, i=$ 3,4 as $F_{3}=w_{3}^{T} \varphi_{3}, F_{4}=w_{4}^{T} \varphi_{4}$. Then $\widehat{F}_{3}=\widehat{w}_{3}^{T} \varphi_{3}$ and $\widehat{F}_{4}=\widehat{w}_{4}^{T} \varphi_{4}$.

Then system (49) can be written as

$$
\begin{aligned}
& \dot{x}_{1}=x_{3}, \\
& \dot{x}_{3}=x_{4}+\widehat{w}_{3}^{T} \varphi_{3}+\widetilde{w}_{3}^{T} \varphi_{3}, \\
& \dot{x}_{4}=x_{2}+\widehat{w}_{4}^{T} \varphi_{4}+\widetilde{w}_{4}^{T} \varphi_{4}, \\
& \dot{x}_{2}=v .
\end{aligned}
$$

Step 4. Choose the nominal system for (51) as

$$
\begin{aligned}
& \dot{x}_{1}=x_{3}, \\
& \dot{x}_{3}=x_{4}, \\
& \dot{x}_{4}=x_{2}, \\
& \dot{x}_{2}=v_{0} .
\end{aligned}
$$


Step 5. Define the sliding surface for nominal system (52) as

$$
s_{0}=x_{1}+3 x_{3}+3 x_{4}+x_{2}
$$

Then

$$
\dot{s}_{0}=\dot{x}_{1}+3 \dot{x}_{3}+3 \dot{x}_{4}+\dot{x}_{2}=x_{3}+3 x_{4}+3 x_{2}+v_{0} .
$$

By choosing

$$
v_{0}=-x_{3}-3 x_{4}-3 x_{2}-k \operatorname{sign}\left(s_{0}\right), \quad k>0,
$$

we have

$$
\dot{s}_{0}=-k \operatorname{sign}\left(s_{0}\right) .
$$

Therefore, nominal system (52) is asymptotically stable.

Step 6. Define the sliding surface for system (51) as

$$
s=s_{0}+z=x_{1}+3 x_{3}+3 x_{4}+x_{2}+z .
$$

Choose $v=v_{0}+v_{s}$.

Then

$$
\begin{aligned}
\dot{s}= & \dot{x}_{1}+3 \dot{x}_{3}+3 \dot{x}_{4}+\dot{x}_{2}+\dot{z} \\
= & x_{3}+3 x_{4}+3 \widehat{w}_{3}^{T} \varphi_{3}+3 \widetilde{w}_{3}^{T} \varphi_{3}+3 x_{2}+3 \widehat{w}_{4}^{T} \varphi_{4} \\
& +3 \widetilde{w}_{4}^{T} \varphi_{4}+v_{0}+v_{s}+\dot{z} .
\end{aligned}
$$

Step 7. The following adaptive laws for $\widetilde{w}_{i}, \widehat{w}_{i}, \quad i=3,4$ and the value of $v_{s}$ are chosen as

$$
\begin{aligned}
\dot{z} & =-x_{3}-3 x_{4}-3 x_{2}-v_{0}, \\
v_{s} & =-3 \widehat{w}_{3}^{T} \varphi_{3}-3 \widehat{w}_{4}^{T} \varphi_{4}-k s, \\
\dot{\widetilde{w}}_{3} & =-3 s \varphi_{3}-k_{1} \widetilde{w}_{3}^{T}, \\
\dot{\widehat{w}}_{3} & =-\dot{\widetilde{w}}_{3}, \\
\dot{\widetilde{w}}_{4} & =-3 s \varphi_{4}-k_{2} \widetilde{w}_{4}^{T}, \\
\dot{\widehat{w}}_{4} & =-\dot{\widetilde{w}}_{4},
\end{aligned}
$$

where $k, k_{1}$ and $k_{2}>0$.

Give

$$
\dot{V}=-k s^{2}-k_{1} \widetilde{w}_{3}^{T} \widetilde{w}_{3}-k_{2} \widetilde{w}_{4}^{T} \widetilde{w}_{4},
$$

where

$$
V=\frac{1}{2} s^{2}+\frac{1}{2} \widetilde{w}_{3}^{T} \widetilde{w}_{3}+\frac{1}{2} \widetilde{w}_{4}^{T} \widetilde{w}_{4}
$$

Choosing

$$
k_{3}=\min \left(k, k_{1}, k_{2}\right) \text {, }
$$

we have

$$
\dot{V} \leq-k_{3}\left(s^{2}+k_{1} \widetilde{w}_{3}^{T} \widetilde{w}_{3}+k_{2} \widetilde{w}_{4}^{T} \widetilde{w}_{4}\right)
$$

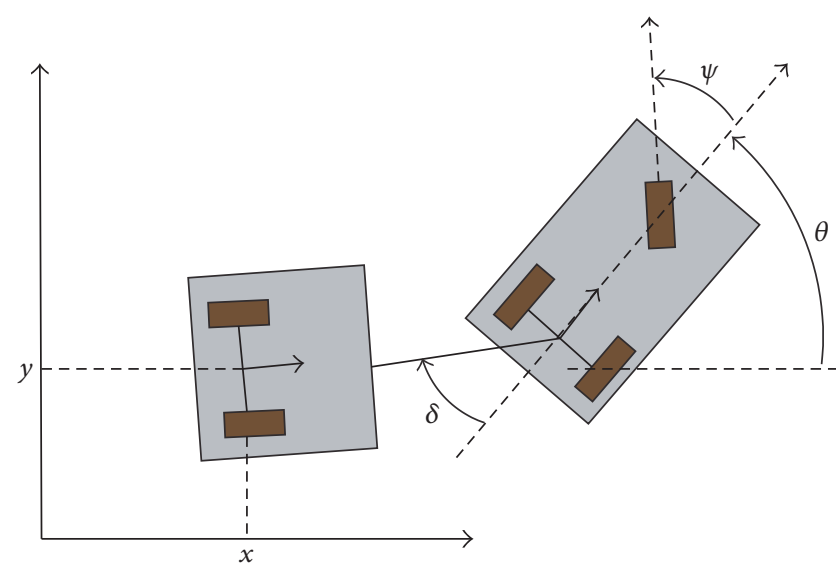

FIGURE 3: The mobile robot with trailer model.

Using the chosen Lyapunov function we can write

$$
\begin{aligned}
& \dot{V} \leq-2 k_{3} V, \\
& \dot{V} \leq-\alpha V^{\beta},
\end{aligned}
$$

where $\alpha=2 k_{3}$ and $\beta=1$.

From this we conclude that $s, \widetilde{w}_{3}, \widetilde{w}_{4} \rightarrow 0$. Since $s \rightarrow 0$, therefore $x \rightarrow 0$.

Simulation results are shown in Figure 5.

4.3. The Mobile Robot with Trailer Model. A car with trailer model, shown in Figure 3, is basically a five-dimensional nonholonomic system having two inputs and five states with depth-one, depth-two, and depth-three Lie brackets. A car with trailer kinematic model [8] can be defined as

$$
\begin{aligned}
& \dot{x}_{1}=\cos x_{3} \cos x_{4} u_{1}, \\
& \dot{x}_{2}=\cos x_{3} \sin x_{4} u_{1}, \\
& \dot{x}_{3}=u_{2}, \\
& \dot{x}_{4}=\frac{1}{l} \sin x_{3} u_{1}, \\
& \dot{x}_{5}=\frac{1}{d} \sin \left(x_{4}-x_{5}\right) \cos x_{3} u_{1} .
\end{aligned}
$$

By assuming $l=d=1$, system (65) can be written in the following standard form:

$$
\dot{x}=G_{1}(x) u_{1}+G_{2}(x) u_{2}, \quad x \in \mathfrak{R}^{5},
$$

where

$$
G_{1}(x)=\left[\begin{array}{c}
\cos x_{3} \cos x_{4} \\
\cos x_{3} \sin x_{4} \\
0 \\
\sin x_{3} \\
\cos x_{3} \sin \left(x_{4}-x_{5}\right)
\end{array}\right] \text {, }
$$




$$
G_{2}(x)=\left[\begin{array}{l}
0 \\
0 \\
1 \\
0 \\
0
\end{array}\right] .
$$

It can be verified that system (66) satisfies the following assumptions which are necessary for steering problem.

(P1) The vectors $G_{i}(x), i=1,2$ are linearly independent and have no singular point for all $x \in M \subseteq \mathfrak{R}^{5}$, where $M$ is some manifold in $\mathfrak{R}^{5}$.

(P2) System (66) satisfies the Lie algebraic rank condition (LARC) for controllability, where the Lie algebra, $L\left(G_{1}, G_{2}\right)(x)$, spans $\mathfrak{R}^{5}$ at each point $x \in M \subseteq \mathfrak{R}^{5}$ : that is, $\operatorname{span}\left(G_{1}(x), G_{2}(x), \ldots, G_{5}(x)\right)=\mathfrak{R}^{5}, \forall x \in$ $M$.

To verify (P1) and (P2), calculate the linearly independent Lie brackets.

$$
\begin{gathered}
G_{3}(x) \stackrel{\text { def }}{=}\left[G_{1}, G_{2}\right](x)=\left[\begin{array}{c}
\sin x_{3} \cos x_{4} \\
\sin x_{3} \sin x_{4} \\
0 \\
-\cos x_{3} \\
\sin x_{3} \sin \left(x_{4}-x_{5}\right)
\end{array}\right], \\
G_{4}(x) \stackrel{\text { def }}{=}\left[G_{1},\left[G_{1}, G_{2}\right]\right](x)=\left[\begin{array}{c}
-\sin x_{4} \\
\cos x_{4} \\
0 \\
0 \\
\cos \left(x_{4}-x_{5}\right)
\end{array}\right],
\end{gathered}
$$

$$
\begin{aligned}
& G_{5}(x) \stackrel{\operatorname{def}}{=}\left[G_{1},\left[G_{1},\left[G_{1}, G_{2}\right]\right]\right](x) \\
&-\sin x_{3} \cos x_{4} \\
&-\sin x_{3} \sin x_{4} \\
& 0 \\
& 0 \\
&=\left[\begin{array}{c} 
\\
-\sin x_{3} \sin \left(x_{4}-x_{5}\right)+\cos x_{3}
\end{array}\right] .
\end{aligned}
$$

If the motion of system is restricted to manifold,

$$
M \stackrel{\text { def }}{=}\left(x \in \Re^{5}:\left|x_{i}\right|<\frac{\pi}{2}, i=3,4\right) .
$$

Then the Lie algebra rank condition, namely, $\operatorname{span}\left(G_{1}(x), G_{2}(x), \ldots, G_{5}(x)\right)=\Re^{5}, \forall x \in M$, is satisfied, hence guaranteeing that system (66) satisfies conditions (P1) and (P2) on the surface $M$.

\subsubsection{Application of the Proposed Algorithm to the Mobile Robot with Trailer Model}

Step 1. System (65) can be written as

$$
\begin{aligned}
& \dot{x}_{1}=\cos x_{3} \cos x_{4} u_{1}, \\
& \dot{x}_{2}=\cos x_{3} \sin x_{4} u_{1}, \\
& \dot{x}_{3}=u_{2}, \\
& \dot{x}_{4}=\sin x_{3} u_{1}, \\
& \dot{x}_{5}=\sin \left(x_{4}-x_{5}\right) \cos x_{3} u_{1} .
\end{aligned}
$$

Step 2. Choose $u_{1}=x_{2} / \cos x_{3} \cos x_{4}$ and $u_{2}=v$.

And, $x_{3}, x_{4} \neq \pi / 2$. Then system (70) can be written as

$$
\begin{aligned}
& \dot{x}_{1}=x_{2}, \\
& \dot{x}_{2}=x_{2} \tan x_{4}, \\
& \dot{x}_{3}=v, \\
& \dot{x}_{4}=x_{2} \tan x_{3} \sec x_{4}, \\
& \dot{x}_{5}=x_{2} \sin \left(x_{4}-x_{5}\right) \sec x_{4} .
\end{aligned}
$$

Step 3. Assume $F_{i}, i=2,4,5$ as uncertainties and let $\widehat{F}_{i}, \quad i=2,4,5$ be an estimate of $F_{i}, \quad i=2,4,5$, respectively. Approximate $F_{i}=w_{i}^{T} \varphi_{i}, \quad i=2,4,5$ and let $\widehat{F}_{i}=\widehat{w}_{i}^{T} \varphi_{i}, \quad i=$ $2,4,5$, respectively. Then system (71) can be written as

$$
\begin{aligned}
& \dot{x}_{1}=x_{2}, \\
& \dot{x}_{2}=x_{4}+\widehat{w}_{2}^{T} \varphi_{2}+\widetilde{w}_{2}^{T} \varphi_{2}, \\
& \dot{x}_{4}=x_{5}+\widehat{w}_{4}^{T} \varphi_{4}+\widetilde{w}_{4}^{T} \varphi_{4}, \\
& \dot{x}_{5}=x_{3}+\widehat{w}_{5}^{T} \varphi_{5}+\widetilde{w}_{5}^{T} \varphi_{5}, \\
& \dot{x}_{3}=v .
\end{aligned}
$$

Step 4. Choose the nominal system for (72) as

$$
\begin{aligned}
& \dot{x}_{1}=x_{2}, \\
& \dot{x}_{2}=x_{4}, \\
& \dot{x}_{4}=x_{5}, \\
& \dot{x}_{5}=x_{3}, \\
& \dot{x}_{3}=v_{0} .
\end{aligned}
$$

Step 5. Define the Hurwitz sliding surface for nominal system (73) as

$$
s_{0}=x_{1}+4 x_{2}+6 x_{4}+4 x_{5}+x_{3} \text {. }
$$

Then

$$
\begin{aligned}
\dot{s}_{0} & =\dot{x}_{1}+4 \dot{x}_{2}+6 \dot{x}_{4}+4 \dot{x}_{5}+\dot{x}_{3} \\
& =x_{2}+4 x_{4}+6 x_{5}+4 x_{3}+v_{0}
\end{aligned}
$$




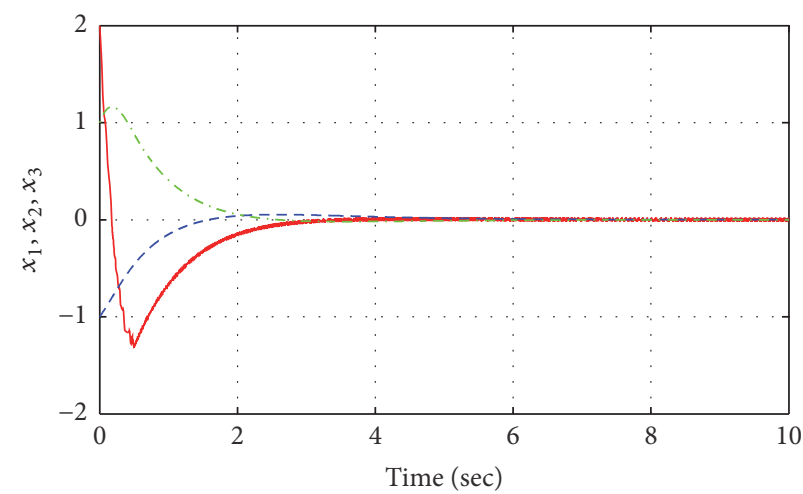

$\begin{array}{ll}- & x_{1} \\ --- & x_{2} \\ -\ldots & x_{3}\end{array}$

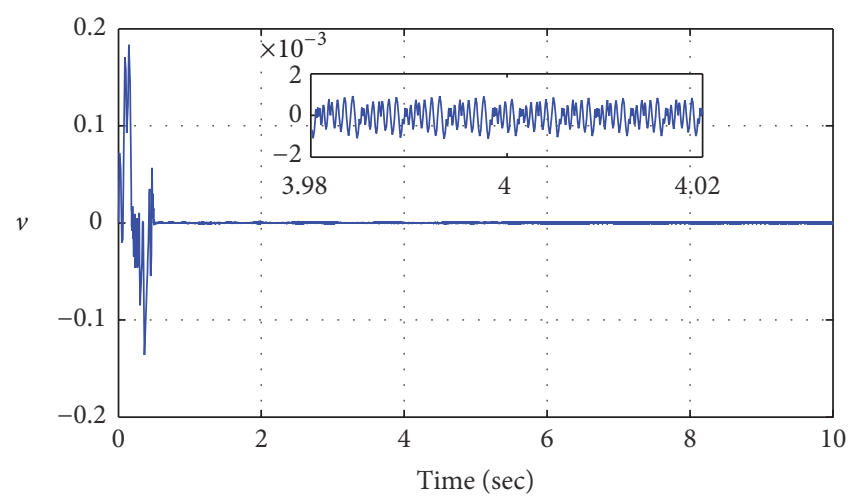

$-v$

(a)

(b)

Figure 4: (a) Time response of the system states corresponding to initial condition $\left(x_{1}(0), x_{2}(0), x_{3}(0)\right)=(2,-1,1)$. (b) Control input $v$.

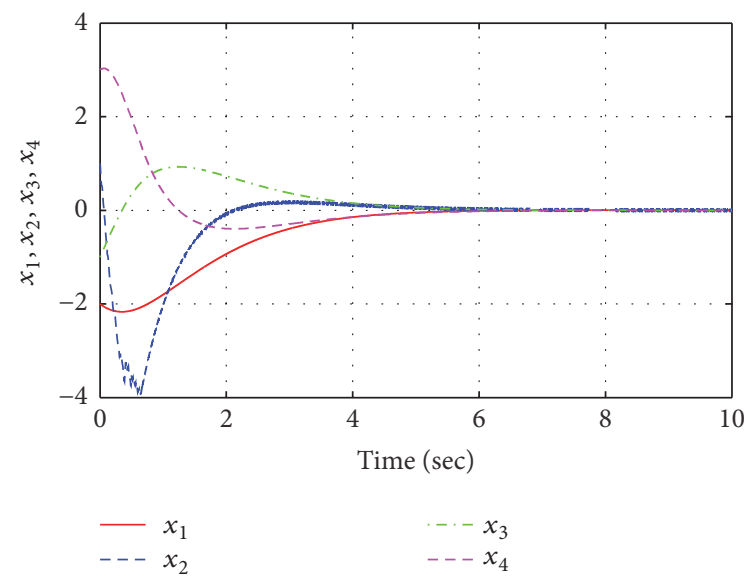

(a)

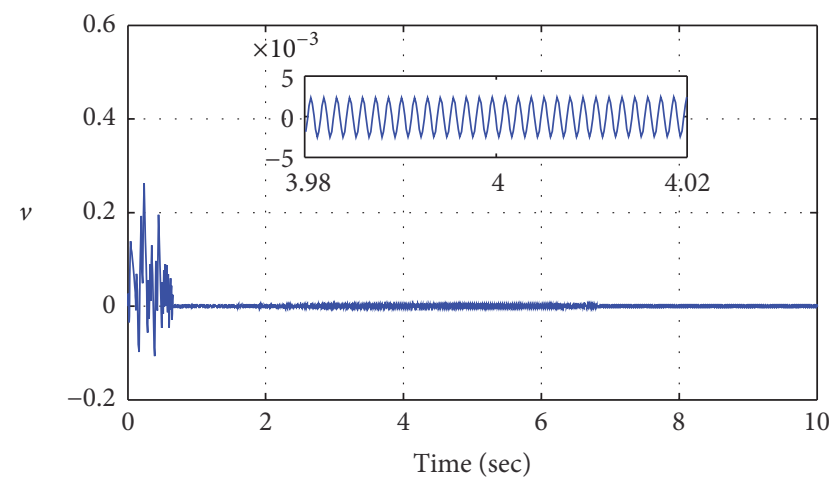

(b)

Figure 5: (a) Time response of the system states corresponding to initial condition $\left(x_{1}(0), \ldots, x_{4}(0)\right)=(-2,1,-1,3)$. (b) Control input $v$.

By choosing

$$
v_{0}=-x_{2}-4 x_{4}-6 x_{5}-4 x_{3}-k \operatorname{sign}\left(s_{0}\right), \quad k>0,
$$

we have

$$
\dot{s}_{0}=-k \operatorname{sign}\left(s_{0}\right) .
$$

Therefore, nominal system (73) is asymptotically stable.

Step 6. Define the sliding surface for system (72) as

$$
s=s_{0}+z=x_{1}+4 x_{2}+6 x_{4}+4 x_{5}+x_{3}+z .
$$

And choose $v=v_{0}+v_{s}$.

$$
\text { Then }
$$

$$
\begin{aligned}
\dot{s}= & \dot{x}_{1}+4 \dot{x}_{2}+6 \dot{x}_{4}+4 \dot{x}_{5}+\dot{x}_{3}+\dot{z} \\
= & x_{2}+4 x_{4}+4 \widehat{w}_{2}^{T} \varphi_{2}+4 \widetilde{w}_{2}^{T} \varphi_{2}+6 x_{5}+6 \widehat{w}_{4}^{T} \varphi_{4} \\
& +6 \widetilde{w}_{4}^{T} \varphi_{4}+4 x_{3}+4 \widehat{w}_{5}^{T} \varphi_{5}+4 \widetilde{w}_{5}^{T} \varphi_{5}+v_{0}+v_{s}+\dot{z} .
\end{aligned}
$$

Step 7. The following adaptive laws for $\widetilde{w}_{i} \& \widehat{w}_{i}$ and the value of $v_{s}$

$$
\begin{aligned}
\dot{z} & =-x_{2}-4 x_{4}-6 x_{5}-4 x_{3}-v_{0}, \\
v_{s} & =-4 \widehat{w}_{2}^{T} \varphi_{2}-6 \widehat{w}_{4}^{T} \varphi_{4}-4 \widehat{w}_{5}^{T} \varphi_{5}-k s, \\
\dot{\widetilde{w}}_{2} & =-4 s \varphi_{2}-k_{1} \widetilde{w}_{2}^{T}, \\
\dot{\widehat{w}}_{2} & \approx-\dot{\widetilde{w}}_{2} \\
\dot{\widetilde{w}}_{4} & =-6 s \varphi_{4}-k_{2} \widetilde{w}_{4}^{T}, \\
\dot{\widehat{w}}_{4} & \approx-\dot{\widetilde{w}}_{4} \\
\dot{\widetilde{w}}_{5} & =-4 s \varphi_{5}-k_{3} \widetilde{w}_{5}^{T}, \\
\dot{\hat{w}}_{5} & \approx-\dot{\tilde{w}}_{5}
\end{aligned}
$$

with $k$ and $k_{i}>0, i=1,2,3$, result in

$$
\dot{V}=-k s^{2}-k_{1} \widetilde{w}_{2}^{T} \widetilde{w}_{2}-k_{2} \widetilde{w}_{4}^{T} \widetilde{w}_{4}-k_{3} \widetilde{w}_{5}^{T} \widetilde{w}_{5},
$$




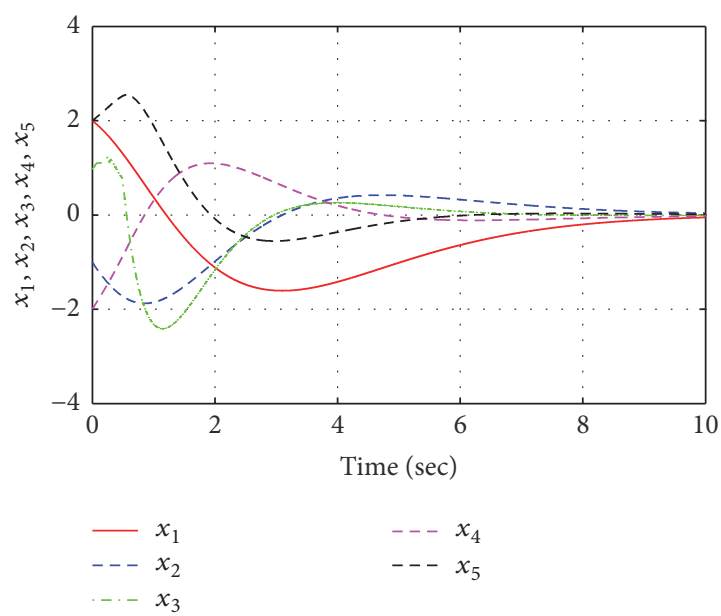

(a)

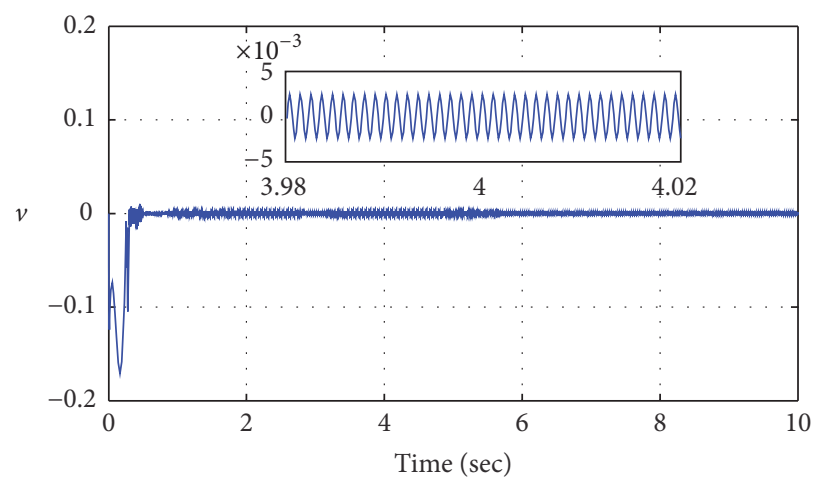

(b)

Figure 6: (a) Time response of the system states corresponding to initial condition $\left(x_{1}(0), \ldots, x_{5}(0)\right)=(2,-1,1,-2,2)$. (b) Control input $v$.

where

$$
V=\frac{1}{2} s^{2}+\frac{1}{2} \widetilde{w}_{2}^{T} \widetilde{w}_{2}+\frac{1}{2} \widetilde{w}_{4}^{T} \widetilde{w}_{4}+\frac{1}{2} \widetilde{w}_{5}^{T} \widetilde{w}_{5}
$$

Choosing

$$
k_{4}=\min \left(k, k_{1}, k_{2}, k_{3}\right) \text {, }
$$

we have

$$
\dot{V} \leq-k_{4}\left(s^{2}+\widetilde{w}_{2}^{T} \widetilde{w}_{2}+\widetilde{w}_{4}^{T} \widetilde{w}_{4}+\widetilde{w}_{5}^{T} \widetilde{w}_{5}\right) .
$$

Using the chosen Lyapunov function we can write

$$
\begin{aligned}
& \dot{V} \leq-2 k_{4} V, \\
& \dot{V} \leq-\alpha V^{\beta},
\end{aligned}
$$

where $\alpha=2 k_{4}$ and $\beta=1$.

From this we conclude that $s, \widetilde{w}_{2}^{T}, \widetilde{w}_{4}^{T}$ and $\widetilde{w}_{5}^{T} \rightarrow 0$. Since $s \rightarrow 0$, therefore $x \rightarrow 0$. Simulation results are shown in Figures 4-6 for different initial conditions.

\section{Simulation Results}

Figures 4(a) and 4(b) show simulation results of the unicycle model and represent that the states and the control effort converge to zero and have settling time of $4 \mathrm{sec}$ and $0.8 \mathrm{sec}$. Figures 5(a) and 5(b) show simulation results of the front wheel car model and represent that the states and the control effort converge to zero and have settling time of $6 \mathrm{sec}$ and $1 \mathrm{sec}$. Figures 6(a) and 6(b) show simulation results for the car with trailer model and represent that the states and control effort converge to zero and have settling time of $10 \mathrm{sec}$ and $0.4 \mathrm{sec}$. Simulation results show the effectiveness of the proposed scheme.

\section{Conclusion}

An adaptive integral sliding mode based control algorithm for the stabilization of nonholonomic drift-free control systems was presented. The objective was to steer the system from any arbitrary initial state to any desired state. The effectiveness of the method was tested on three different nonholonomic driftfree systems: the unicycle model, the front wheel car model, and the mobile robot with trailer model. The aim was to steer the systems to a desired value which was assumed to be zero. It is evident from the simulation results that the objective has been achieved. This method is general and can be employed to steer a variety of mechanical systems with nonholonomic constraints.

\section{Competing Interests}

The authors declare that there is no conflict of interests regarding the publication of this paper.

\section{References}

[1] R. W. Brockett, "Asymptotic stability and feedback stabilization," in Differential Geometric Control Theory, R. S. Millman and H. J. Sussmann, Eds., vol. 27, pp. 2961-2963, Birkhäauser, Boston, Mass, USA, 1983.

[2] R. M. Murray, G. Walsh, and S. S. Sastry, "Stabilization and tracking for nonholonomic control systems using time-varying state feedback," in Proceedings of the IFAC Symposium on Nonlinear Control Systems Design 1992, pp. 109-114, June 1992.

[3] A. Astolfi, "Discontinuous control of nonholonomic systems," Systems and Control Letters, vol. 27, no. 1, pp. 37-45, 1996.

[4] Y. Liu and H. Yu, "A survey of underactuated mechanical systems," IET Control Theory and Applications, vol. 7, no. 7, pp. 921-935, 2013.

[5] S. K. Shah and H. G. Tanner, "Control of stochastic unicycletype robots," in Proceedings of the IEEE International Conference on Robotics and Automation (ICRA '15), pp. 389-394, May 2015.

[6] R. M. Murray and S. S. Sastry, "Nonholonomic motion planning: steering using sinusoids," IEEE Transactions on Automatic Control, vol. 38, no. 5, pp. 700-716, 1993.

[7] S. S. Ge, Z. Wang, and T. H. Lee, "Adaptive stabilization of uncertain nonholonomic systems by state and output feedback," Automatica, vol. 39, no. 8, pp. 1451-1460, 2003. 
[8] W. Pasillas-Lepine and W. Respondek, "Conversion of the kinematics of the $n$-trailer system into Kumpera-Ruiz normal form and motion planning through the singular locus," in Proceedings of the 38th IEEE Conference on Decision and Control (CDC '99), vol. 3, pp. 2914-2919, Phoenix, Ariz, USA, December 1999.

[9] V. I. Utkin, Sliding Modes in Control and Optimization, Springer Science \& Business Media, Berlin, Germany, 2013.

[10] Z. Xi, G. Feng, Z. P. Jiang, and D. Cheng, "Output feedback exponential stabilization of uncertain chained systems," Journal of the Franklin Institute, vol. 344, no. 1, pp. 36-57, 2007.

[11] A. Donaire, J. G. Romero, T. Perez, and R. Ortega, "Smooth stabilisation of nonholonomic robots subject to disturbances," in Proceedings of the IEEE International Conference on Robotics and Automation (ICRA '15), pp. 4385-4390, IEEE, Seattle, Wash, USA, May 2015.

[12] C. Gruber and M. Hofbaur, "Remarks on the classification of wheeled mobile robots," Mechanical Sciences, vol. 7, no. 1, pp. 93-105, 2016.

[13] J. Zhang and Y. Liu, "Adaptive stabilization of a class of highorder uncertain nonholonomic systems with unknown control coefficients," International Journal of Adaptive Control and Signal Processing, vol. 27, no. 5, pp. 368-385, 2013.

[14] L.-Y. Sun, S. Tong, and Y. Liu, "Adaptive backstepping sliding mode $H_{\infty}$ control of static var compensator," IEEE Transactions on Control Systems Technology, vol. 19, no. 5, pp. 1178-1185, 2011.

[15] D. Liberzon, Switching in Systems and Control, Springer Science \& Business Media, 2012.

[16] S. Mobayen, "Finite-time tracking control of chained-form nonholonomic systems with external disturbances based on recursive terminal sliding mode method," Nonlinear Dynamics, vol. 80, no. 1-2, pp. 669-683, 2015.

[17] J.-M. Yang and J.-H. Kim, "Sliding mode control for trajectory tracking of nonholonomic wheeled mobile robots," IEEE Transactions on Robotics and Automation, vol. 15, no. 3, pp. 578-587, 1999.

[18] S. Ding, J. Wang, and W. X. Zheng, "Second-order sliding mode control for nonlinear uncertain systems bounded by positive functions," IEEE Transactions on Industrial Electronics, vol. 62, no. 9, pp. 5899-5909, 2015.

[19] Y. Wu, F. Gao, and Z. Zhang, "Saturated finite-time stabilization of uncertain nonholonomic systems in feedforward-like form and its application," Nonlinear Dynamics, vol. 84, no. 3, pp. 1609-1622, 2016.

[20] S. Ding, A. Levant, and S. Li, "Simple homogeneous slidingmode controller," Automatica, vol. 67, pp. 22-32, 2016.

[21] H. Chen, B. Li, B. Zhang, and L. Zhang, "Global finite-time partial stabilization for a class of nonholonomic mobile robots subject to input saturation," International Journal of Advanced Robotic Systems, vol. 12, no. 11, article 159, 2015.

[22] Y. Wu, F. Gao, and Z. Liu, "Finite-time state-feedback stabilisation of non-holonomic systems with low-order non-linearities," IET Control Theory \& Applications, vol. 9, no. 10, pp. 1553-1560, 2015.

[23] F. Gao and F. Yuan, "Adaptive finite-time stabilization for a class of uncertain high order nonholonomic systems," ISA Transactions, vol. 54, pp. 75-82, 2015.

[24] F. Gao, Y. Wu, and Z. Zhang, "Finite-time stabilization of uncertain nonholonomic systems in feedforward-like form by output feedback," ISA Transactions, vol. 59, pp. 125-132, 2015.
[25] M. Defoort, T. Floquet, A. Kokosy, and W. Perruquetti, "Integral sliding mode control for trajectory tracking of a unicycle type mobile robot," Integrated Computer-Aided Engineering, vol. 13, no. 3, pp. 277-288, 2006.

[26] Z. Tang, J. Zhou, X. Bian, and H. Jia, "Simulation of optimal integral sliding mode controller for the depth control of AUV," in Proceedings of the IEEE International Conference on Information and Automation (ICIA '10), pp. 2379-2383, IEEE, Harbin, China, June 2010.

[27] A. C. Huang, Y. F. Chen, and C. Y. Kai, Adaptive Control of Underactuated Mechanical Systems, World Scientific, 2015. 


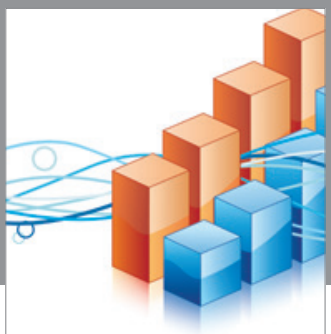

Advances in

Operations Research

vatem alat4

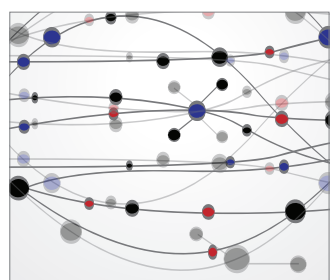

\section{The Scientific} World Journal
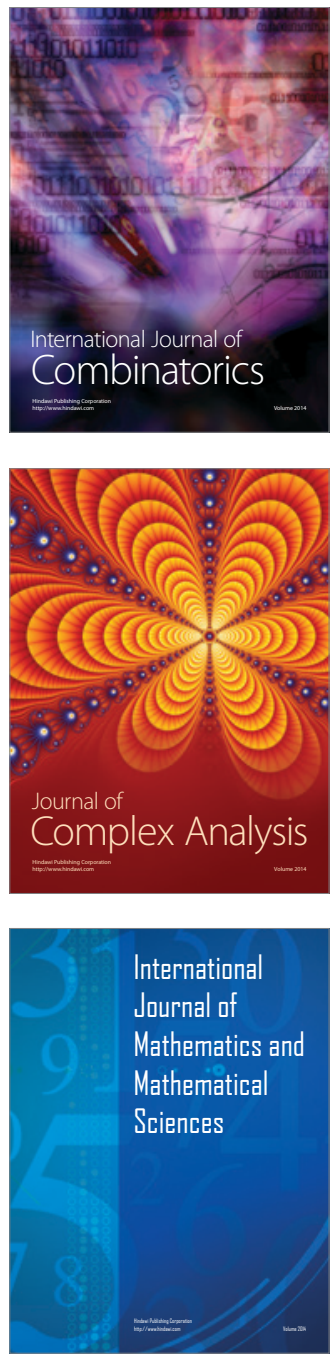
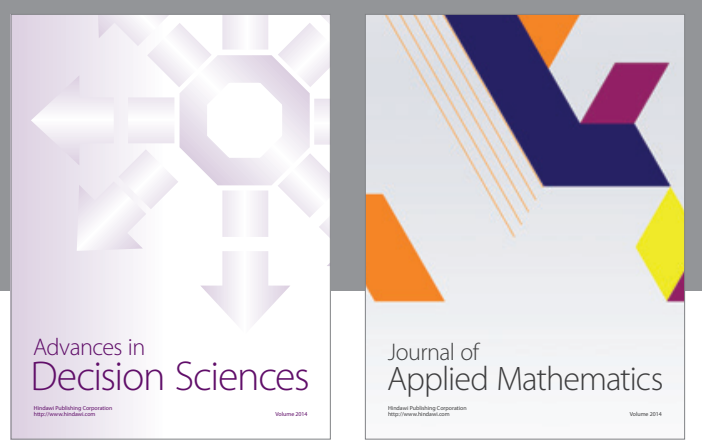

Algebra

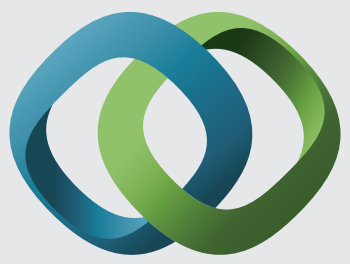

\section{Hindawi}

Submit your manuscripts at

http://www.hindawi.com
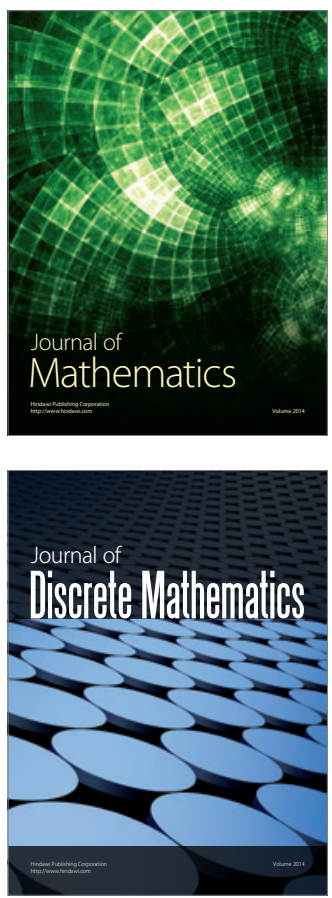

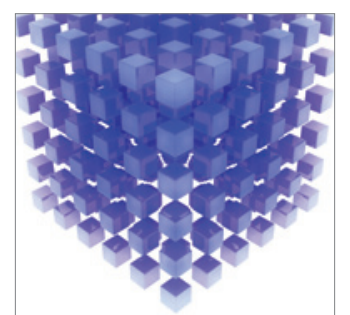

Mathematical Problems in Engineering
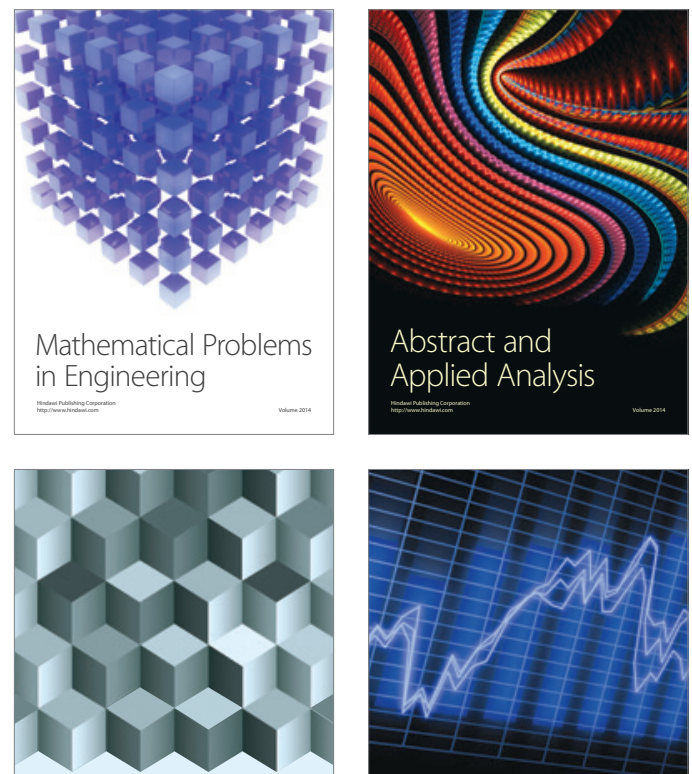

Journal of

Function Spaces

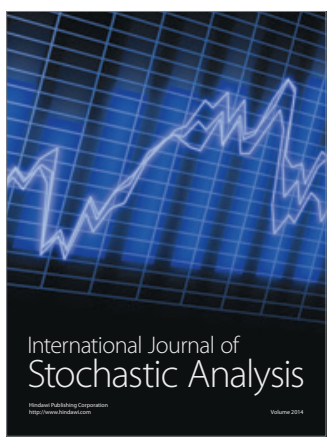

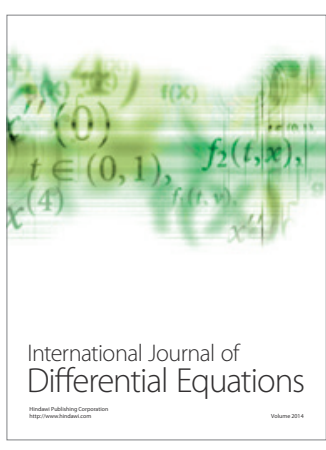
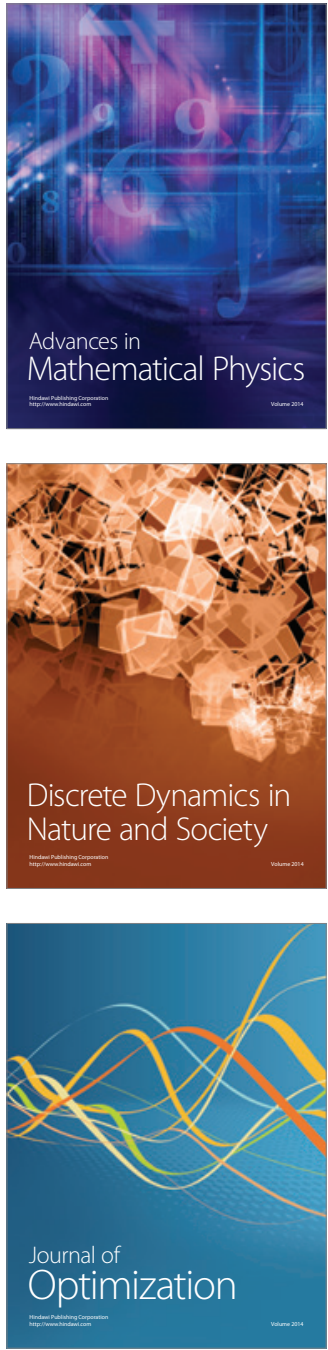